\title{
Comparative Morphological Study on the Embryonic and Neonatal Development of the Filiform Papillae and Teeth in Mice
}

\author{
Soon-Jeong Jeong ${ }^{1,2, \dagger}$ \\ 'Department of Dental Hygiene, College of Health Science, ${ }^{2}$ Institute of Basic Science for Well-aging, Youngsan \\ University, Yangsan 50510, Korea
}

\begin{abstract}
Background: In the early stages of development, teeth and lingual papillae are induced and developed through special and complex epithelial-mesenchymal interactions. Tooth completion indicates the beginning of the weaning phase, and accordingly, many oral tissues and organs are completed, and it is thought that their developmental completion times are related to each other. The purpose of this study was to clarify the embryonic and neonatal development of the filiform papillae and mandibular molar tooth, and discuss the developmental relationship between these organs by comparing the developmental completion times.

Methods: Embryos at embryonic day 15 (EM15), 17 (EM17), and 21 (EM21) and mice at neonatal day 1 (NE1), 5 (NE5), 10 (NE10), and 21 (NE21) were used for experimentation. Tissues dissected from embryos and mice were fixed, and processed for histological analysis. Sections from the tissues were stained with hematoxylin and eosin for observation under a light microscope. Results: Based on the histological analysis results, the developmental process of the lingual epithelium covering the dorsal surface of the tongue was classified into three stages: initiation, morphogenesis, and functional. The development of the filiform papillae begins at EM17; undergoes rapid morphological changes in epithelial cells at EM21, PN1 and PN5, and reaches the functional stage at PN10, which is the sucking phase. Tooth development begins at EM13 or 15 and is completed at NE21 through prenatal and postnatal development.

Conclusion: The development of the filiform papillae was initiated late and completed quickly through embryonic and neonatal development in comparison with the mandibular molar tooth. The filiform papillae are considered to play an important role in sucking rather than mastication as it is completed in the sucking phase.
\end{abstract}

Key Words: Embryonic development, Filiform papillae, Mouse, Neonatal development, Tooth

\section{Introduction}

During development, the mouse tongue is divided into oral and pharyngeal sections by the terminal sulcus and its dorsal surface is covered with lingual papillae ${ }^{1)}$. The lingual papillae perform various functions during mastication, transportation and swallowing of food ${ }^{2}$, as well as have a barrier function that prevents the penetration of harmful substances such as bacteria ${ }^{3)}$. On the dorsal surface of the tongue, four types of lingual papillae are found: circum- vallate, fungiform, foliate, and filiform, each of which is distributed on the surface of the tongue according to specific patterns (Fig. 1) ${ }^{1,4,5)}$. Based on the histological analysis of the presence or absence of taste buds, the lingual papillae are divided into gustatory papillae, which plays an important role in the gustatory system, and mechanical papillae, which plays a role in mastication ${ }^{3)}$. Circumvallate, fungiform and foliate papillae with taste buds belong to the gustatory papillae and filiform papillae without taste buds belong to the mechanical papillae ${ }^{3)}$. In 
A

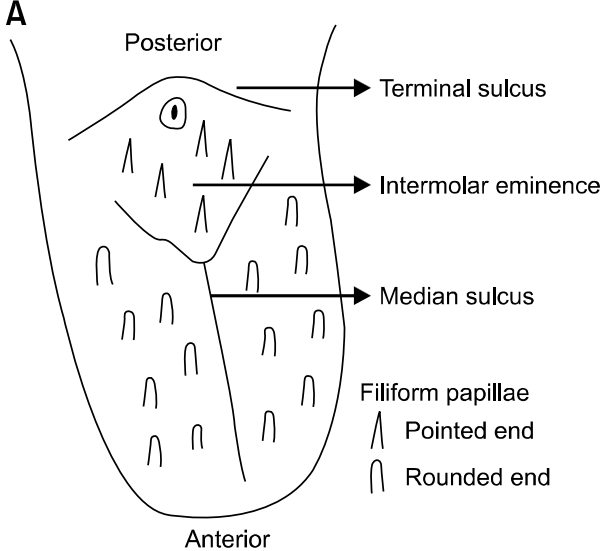

B

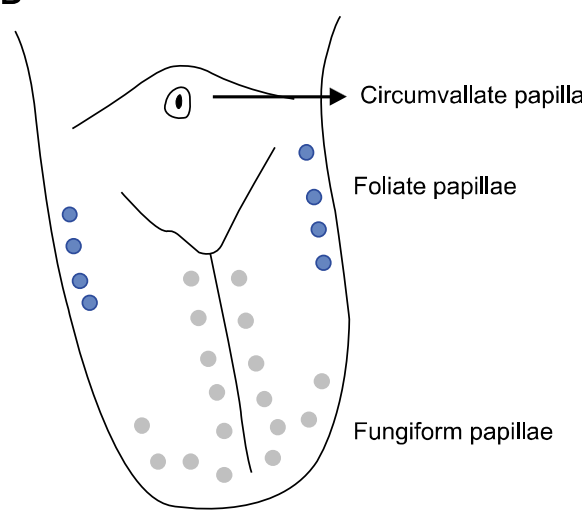

Fig. 1. Schematic diagram of lingual papilla distributed on the dorsal surface of the tongue. (A) Filiform papillae, (B) circumvallate papilla, foliate papillae and fungiform papillae. mice, one dome-shaped circumvallate papilla with keratinized epithelium is located in the center of the terminal sulcus $^{6,7)}$, the fungiform papillae have a single taste bud in the apical portion of the thinly keratinized or nonkeratinized epithelium $^{8)}$, and the foliate papillae with red leaf-like ridges are variable in size and shape and have many taste buds in the non-keratinized epithelium ${ }^{9}$. Filiform papillae, the most common and cone-shaped of the lingual papillae are divided into two types according to the shape of the tip and the location of distribution ${ }^{3)}$. The filiform papillae located in the intermolar eminence have a pointed end while those located in other regions have a rounded end ${ }^{3)}$. It is not yet known which of the developmental processes determines the morphological difference between filiform papillae. Recently, many studies of tongue papillae have been conducted and reported in various ways using protein expression, signaling pathways, molecular mechanisms, and tissue interactions but have mainly been about the gustatory papillae and taste buds $^{1,10-12)}$. To date, filiform papillae development remains unclear $^{13-15)}$.

In the early stages of development, teeth and lingual papillae are induced and developed through special and complex epithelial-mesenchymal interactions ${ }^{16,17)}$. Organs that are induced and formed by epithelial-mesenchymal interactions include the salivary and mammary glands, hairs, and feathers, and these organs have similarities in their initiation and formation processes, although their morphological, anatomical, and physiological properties are significantly different ${ }^{1,18)}$. The process of tooth development is well known and classified into the bud, cap, bell, crown, and functional stages according to the morphological characteristics ${ }^{19,20)}$, and is completed through prenatal and postnatal development ${ }^{20)}$. The completion of the teeth marks the beginning of the weaning phase, and accordingly, many oral tissues and organs are completed, and it is believed that their developmental completion time will be related to each other.

The purpose of this study was to clarify the embryonic and neonatal development of the filiform papillae and mandibular molar tooth, and discuss the developmental relationship between these organs by comparing the developmental completion times.

\section{Materials and Methods}

Animal maintenance and sacrifice for experiments were conducted in accordance with the guidelines of Youngsan University for the care and use of animals in research.

\section{Animals}

Embryos and mice were obtained from ICR outbred mice. Embryos at embryonic day 15 (EM15), 17 (EM17) and 21 (EM21) and mice at neonatal day 1 (NE1), 5 (NE5), 10 (NE10) and 21 (NE21) were used for the experiments. Tissues dissected from embryos and mice were washed with cold phosphate-buffered saline (PBS), then fixed with cold $4 \%$ paraformaldehyde in PBS, and processed for histological analysis.

\section{Histological analysis}

The fixed tissues were decalcified with $10 \%$ ethylenedi- 
aminetetraacetic acid for 4 weeks. After washing the decalcifying solution from the tissues, dehydration was performed with a series of graded ethyl alcohols. The dehydrated tissues were treated with xylene and then embedded in paraffin wax. The tissues were cut to a thickness of $6 \sim 7 \mu \mathrm{m}$ and sections were stained with hematoxylin and eosin for observation with a light microscope.

\section{Results}

\section{Embryonic development}

\section{1) Embryonic day 15 (EM15)}

The lingual epithelium covering the dorsal surface of the tongue, which is thought to occur as filiform papillae at EM15, is observed as striated cuboidal epithelium cells (Fig. 2A). Microvilli were observed on the surface of cuboidal epithelial cells and showed characteristics before development began (Fig. 2B). Under the lingual epithelium,
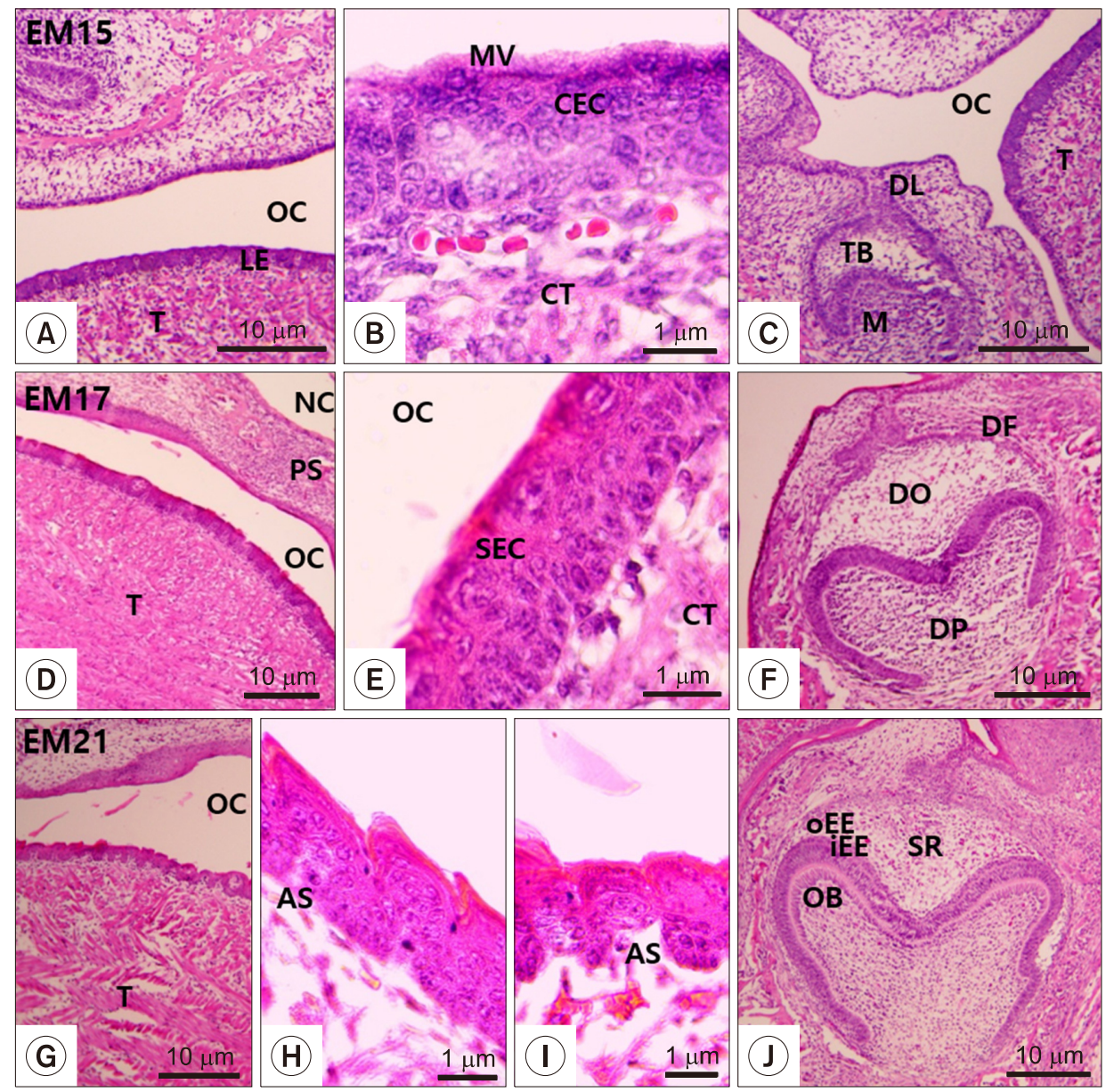

Fig. 2. Light micrographs showing embryonic development of filiform papillae and tooth in coronal section of mice head. (A C) EM15; (D F) EM17; (G J) EM21. (A) LE of the dorsal surface of the T in EM 15. (B) Higher magnified micrograph of stratified CEC covered with MV. (C) Mandibular molar tooth of late bud stage in EM15. (D) Elongated and thickened LE in EM 17. (E) Higher magnified micrograph of the MV disappeared and the elongated and thickened stratified squamous epithelium in EM17. (F) Molar tooth with typical cap stage characteristics in EM17. (G) More compacted LE of EM21. (H) Higher magnified micrograph of LE showing the onset of development into pointed end filiform papillae in EM21. (I) Higher magnified micrograph of LE showing the onset of development into rounded end filiform papillae in EM 21. (J) Developing mandibular molar tooth of early bell stage in EM21. AS: archlike structure, CEC: cuboidal epithelial cells, CT: connective tissue, DF: dental follicle, DL: dental lamina, DP: dental papilla, DO: dental organ, iEE: inner enamel epithelial cells, LE: lingual epithelium, M: mesenchyme, MV: microvilli, NC: nasal cavity, OB: odontoblasts, OC: oral cavity, oEE: outer enamel epithelial cells, PS: palatal shelf, SEC: squamous epithelial cell, SR: stellate reticulum cells, T: tongue, TB: tooth bud. 

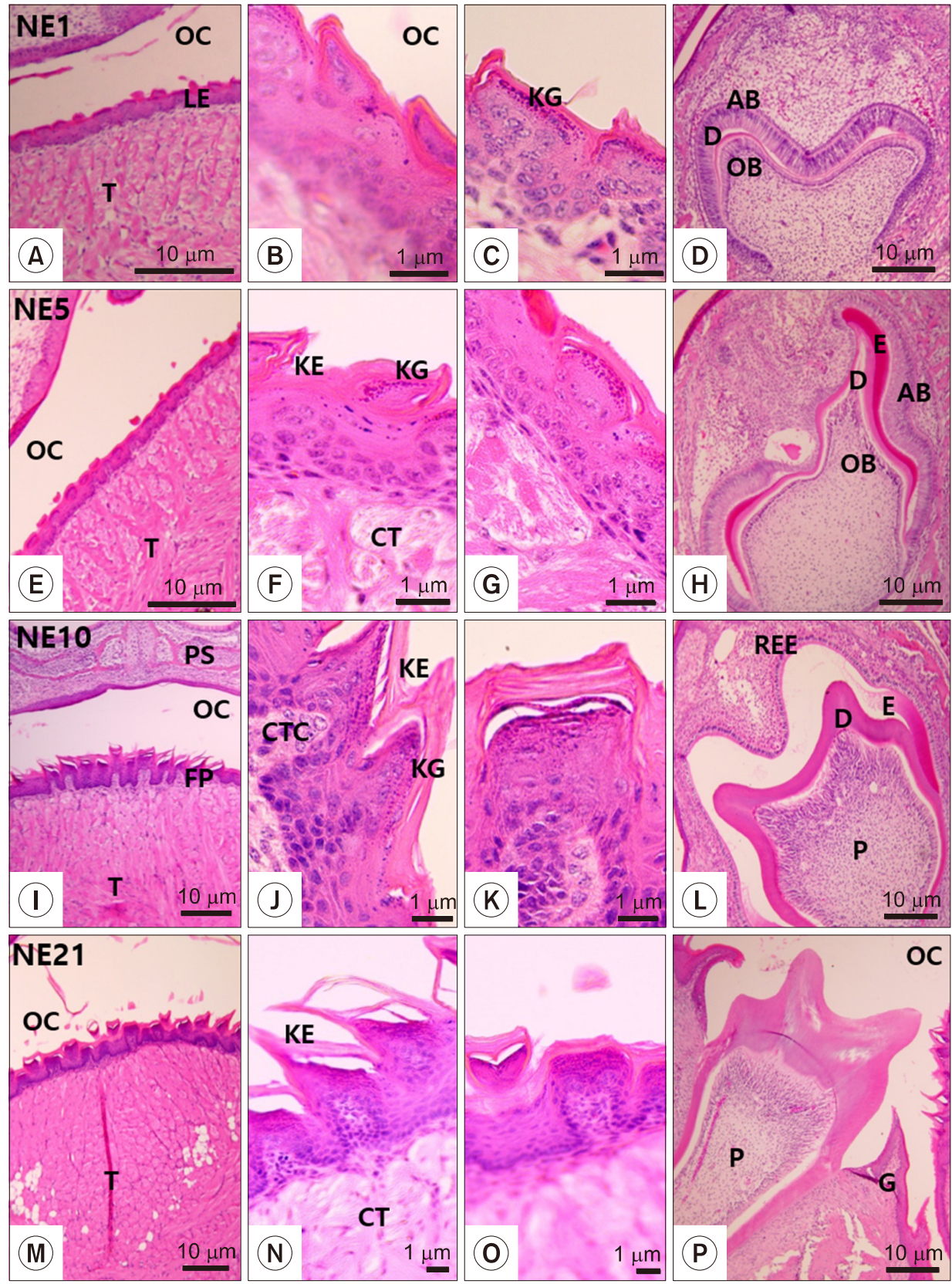

Fig. 3. Light micrographs showing neonatal development of filiform papillae and tooth in coronal section of mice head. (A $\sim D)$ NE1; $(E \sim H)$ NE5; (I L) NE10; (M P) NE21. (A) LE with distinct FP in NE1. (B) Higher magnified micrograph of pointed end FP. (C) Higher magnified micrograph of rounded end FP with KG on surface of epithelium. (D) Molar tooth of the mandibles of NE1 showing a more advanced bell stage characteristic. (E) LE of NE5 showing a form similar to that of NE1. Higher magnified micrograph of pointed end (F) and rounded end (G) FP with numerous KG of NE5. (H) Mandibular molar tooth showing the characteristics of late advanced bell stage. (I) Matured FP with keratinized stratified squamous epithelium in NE10. Higher magnified micrograph of matured pointed end $(\mathrm{J})$ and rounded ends (K) FP of NE10 with numerous KG and a keratinized layer that looks like a thick, bundle of threads on the surface and a well-developed CTC at the bottom. (L) Mandibular molar tooth of NE10 showing typical crown stage characteristics. (M) FP of NE21 showing a form similar to that of NE10. Higher magnified micrograph of mature pointed end (N) and rounded ends (O) FP of NE21 showing a form similar to that of NE10. (P) Molar tooth of functional stage erupted into the oral cavity in NE21. AB: ameloblast, CT: connective tissue, CTC: connective tissue core, D: dentin, E: enamel, FP: filiform papillae, G: gingiva, KE: keratinized epithelium, KG: keratohyalin granules, LE: lingual epithelium, OC: oral cavity, OB: odontoblasts, P: pulp, PS: palatal shelf, REE: reduced enamel epithelium, T: tongue. 
a single layer of mesenchymal cells was observed (Fig. 2B).

The developing mandibular molar teeth at EM15 were characterized by the late bud stage, with the dental lamina formed from the primary epithelial band toward the mesenchyme with the dental organ and dental papilla differentiated from the tooth bud (Fig. 2C).

\section{2) Embryonic day 17 (EM17)}

On EM 17, the microvilli disappeared and the elongated and thickened lingual epithelium was observed on the dorsal surface of the tongue, confirming that the development of the filiform papillae was initiated and showed a typical striated squamous epithelium composed of cuboidal cells in the basal layer and squamous cells in the apical cell layers, but characteristic changes such as archlike structures were not confirmed (Fig. 2E).

The molar tissue at EM17 was divided into complete dental organ, dental papilla and dental follicle, and showed typical cap stage characteristics (Fig. 2F).

\section{3) Embryonic day 21 (EM21)}

The lingual epithelium at EM21 was more compact (Fig. 2G). The morphological changes of the surface and the development of filiform papillae with pointed and rounded ends began (Fig. 2H, 2I). Archlike structures that; migrated upward of the condensed mesenchymal cells located below the epithelium; were observed (Fig. 2H, 2I).

The developing mandibular molar tissue at EM21 was characterized by the early bell stage (Fig. 2J). That is, the cells constituting the dental organs differentiated into outer enamel epithelial cells, stellate reticulum cells, stratum intermedium cells, and inner enamel epithelial cells. Dental papilla cells in contact with the inner enamel epithelial cells differentiated from cuboidal cells to columnar cells and were observed as presecretory odontoblasts (Fig. 2J).

\section{Neonatal development}

\section{1) Neonatal day 1 (NE1)}

At NE1, a clearly distinguished striated squamous epithelium of filiform papillae with connective tissue located below it was observed (Fig. 3A). Moreover, keratohyaline granules responsible for morphogenesis of the keratinized layer were found on the surface of the filiform papillae with rounded ends (Fig. 3C), unlike the epithelium of the filiform papillae with pointed ends (Fig. 3B).

The molar tissues of the mandibles at NE1 exhibited a more advanced bell stage characterized by the dentine and predentin layers formed by odontoblasts along with well-differentiated ameloblasts and odontoblasts (Fig. 3D).

\section{2) Neonatal day 5 (NE5)}

At NE5, the filiform papillae and connective tissue located below it were similar to that of at NE1, but they were more developed (Fig. 3E). Numerous keratohyaline granules were observed in both pointed- (Fig. 3F) and rounded-end (Fig. 3G) filiform papillae.

The developing mandibular molar tissue observed at NE5 has long and forming thick enamel, predentin, and dentine layers, and well-developed secretory ameloblasts, showing the characteristics of a late advanced bell stage (Fig. 3H).

\section{3) Neonatal day 10 (NE10)}

Filiform papillae with a keratinized stratified squamous epithelium and irregular connective tissue thought to be mature structures, were observed at NE10 (Fig. 3I). Namely, filiform papillae with pointed and rounded ends had numerous keratohyaline granules on the superficial layers, a thick keratinized layer which looked like a bundle of threads, and a well-developed connective tissue core beneath the keratinized layer (Fig. 3J, 3K).

The molar tissue at NE10 had thick, well-developed enamel and dentine layers, but ameloblasts that shrank and lost function to become reduced enamel epithelium were observed, showing typical crown stage characteristics (Fig. 3L).

\section{4) Neonatal day 21 (NE21)}

The filiform papillae at NE21 were a matured form with no morphological differences from NE10 (Fig. 3M 3O).

At NE21, the mandibular molar tooth erupted into the 
Table 1. Comparison of Embryonic and Neonatal Development of Filiform Papillae and Tooth

\begin{tabular}{lllll}
\hline \multicolumn{2}{c}{ Developmental timing } & \multicolumn{1}{c}{ Lingual papillae } & \multicolumn{1}{c}{ Tooth } & Phase \\
\hline Embryonic development & EM13 & Developmental Initiation of fungiform papillae & & Embryonic phase \\
& EM14 & & & \\
& EM15 & Developmental Initiation of filiform papillae ${ }^{28)}$ & Bud stage & Cap stage \\
& EM17 & Initiation stage of filiform papillae & Early bell stage & Sucking phase \\
& EM21 & Morphogenesis stage of filiform papillae & Bell stage & Late advanced bell stage \\
& NE1 & Morphogenesis of filiform papillae & Crown stage & Weaning phase ${ }^{19,20)}$ \\
\hline
\end{tabular}

oral cavity and was at a functional stage (Fig. 3P).

\section{Discussion}

The development of oral tissues and organs, including the lingual papillae and teeth, begins very early in embryonic development, which is sensitive to genetic and epigenetic effects ${ }^{8)}$. The lingual papillae and teeth begin to develop, and the tissues are formed through epithelialmesenchymal interactions ${ }^{16,18)}$. Despite their structural, anatomical, physiological and functional differences, it is known that their development processes have many similarities ${ }^{1,16)}$. Based on histological analysis results, the developmental process of the lingual epithelium for filiform papillae covering the dorsal surface of the tongue was classified into three stages: initiation, morphogenesis, and functional (Table 1). In the early stages of embryonic development, a characteristic of undifferentiated epithelial cells is the presence of microvilli on the surface of the epithelial cells, and when differentiation of the epithelial cells begins, the microvilli disappear, ${ }^{2,21-23)}$. Based on this, EM17 was identified as the initiation stage of the development of filiform papillae. And then, rapid morphological changes in epithelial cells have been observed and this period is divided into morphogenesis stages. That is, the surface of the epithelium at EM21, PN1 and PN5 were clearly distinguished from the prominent papillae with pointed and rounded ends, and keratin granules were observed. The filiform papillae at PN10 reached the functional stage with well-developed keratohyalin granules and keratinized layers. Namely, pointed- and rounded-end filiform papillae were completed at PN10. Recently, it has been reported that the expression of Shh, Ptc, Gli-1, Bmp-4, and Fgf-8 signaling molecules had been confirmed in the embryonic developmental process of mouse lingual papillae, and they play an important role for morphogenesis in the initiation stage of embryonic development ${ }^{24,25)}$. Although there are no direct results on inducing and determining morphological differences between pointed- and rounded-end filiform papillae, Bmp-4 and Shh expression are thought to be particularly relevant ${ }^{1)}$. In contrast, there are reports that the developmental initiation of fungiform papillae occurs at EM13 or EM14 ${ }^{3,26)}$, and taste buds in the fungiform papillae and teeth are completed at NE21 which is the weaning phase ${ }^{19,27)}$. The development of the filiform papillae initiates at EM15 ${ }^{28)}$ and is completed before birth ${ }^{29,30)}$. However, in this study, the development of the filiform papillae initiated at EM17 and reached the functional stage at PN10 which is the sucking phase. The development of the filiform papillae initiated late and completed quickly in comparison with the fungiform papillae and teeth, and is completed through embryonic and neonatal development such as the tooth and salivary gland formed as a result of epithelialmesenchymal interactions (Table 1). Although filiform papillae are known to maintain food in the oral cavity, increase the contact and friction surfaces between the tongue and food, mastication, swallowing, sucking ${ }^{2,9,31,32)}$, they are considered to play an important role in sucking rather than mastication as it is completed in the sucking phase. 


\section{Notes}

\section{Conflict of interest}

No potential conflict of interest relevant to this article was reported.

\section{Ethical approval}

This article is not necessary for IRB screening because it is an experimental paper using animal.

\section{Author contributions}

Conceptualization, Data acquisition, Formal analysis, Funding, Supervision, Writing-original draft, Writing-review \& editing: Soon-Jeong Jeong.

\section{ORCID}

Soon-Jeong Jeong, https://orcid.org/0000-0002-8959-4663

\section{Acknowledgements}

This study was supported by Youngsan University Research Fund of 2019.

\section{References}

1. Kim JY, Mochizuki T, Akita K, Jung HS: Morphological evidence of the importance of epithelial tissue during mouse tongue development. Exp Cell Res 290: 217-226, 2003. https://doi.org/10.1016/s0014-4827(03)00319-7

2. Skieresz-Szewczyk K, Jackowiak H: Development of mechanical papillae of the tongue in the domestic goose (Anser anser f. domestica) during the embryonic period. Protoplasma 254: 147-160, 2017. https://doi.org/10.1007/s00709-015-0927-x

3. Kawasaki K, Porntaveetus T, Oommen S, et al.: Bmp signalling in filiform tongue papillae development. Arch Oral Biol 57: 805-813, 2012. https://doi.org/10.1016/j.archoralbio.2011.11.014

4. Mistretta CM: Developmental neurobiology of the taste system. In: Getchell TV, Doty RL, Bartoshuk L, Snow JB, eds. Smell and taste in health and disease. Raven Press, New York, pp.35-64, 1991.

5. Iwasaki S, Yoshizawa H, Kawahara I: Ultrastructural study of the relationship between the morphogenesis of filiform papillae and the keratinisation of the lingual epithelium in the rat. J Anat 195(Pt 1): 27-38, 1999.

https://doi.org/10.1046/j.1469-7580.1999.19510027.x

6. Shindo J, Yoshimura K, Kobayashi K: Comparative morphological study on the stereo-structure of the lingual papillae and their connective tissue cores of the American beaver (Castor canadensis). Okajimas Folia Anat Jpn 82: 127-138, 2006. https://doi.org/10.2535/ofaj.82.127

7. Rothova M, Thompson H, Lickert H, Tucker AS: Lineage tracing of the endoderm during oral development. Dev Dyn 241: 1183-1191, 2012. https://doi.org/10.1002/dvdy.23804

8. Mistretta CM, Liu HX: Development of fungiform papillae: patterned lingual gustatory organs. Arch Histol Cytol 69: 199-208, 2006. https://doi.org/10.1679/aohc.69.199

9. Standring $S$ : Neck and upper aerodigestive traect. In: Standring S, Gray H, eds. Gray's anatomy: the anatomical basis of clinical practice. 40th ed. Churchill Livingstone/ Elsevier, Edinburgh, pp.50-72, 2008.

10. Jitpukdeebodintra S, Chai Y, Snead ML: Developmental patterning of the circumvallate papilla. Int J Dev Biol 46: 755-763, 2002.

11. Hall JM, Bell ML, Finger TE: Disruption of sonic hedgehog signaling alters growth and patterning of lingual taste papillae. Dev Biol 255: 263-277, 2003. https://doi.org/10.1016/s0012-1606(02)00048-9

12. Kim JY, Lee MJ, Cho KW, et al.: Shh and ROCK1 modulate the dynamic epithelial morphogenesis in circumvallate papilla development. Dev Biol 325: 273-280, 2009. https://doi.org/10.1016/j.ydbio.2008.10.034

13. Beites CL, Hollenbeck PL, Kim J, Lovell-Badge R, Lander $\mathrm{AD}$, Calof AL: Follistatin modulates a BMP autoregulatory loop to control the size and patterning of sensory domains in the developing tongue. Development 136: 2187-2197, 2009. https://doi.org/10.1242/dev.030544

14. Okubo T, Pevny LH, Hogan BL: Sox2 is required for development of taste bud sensory cells. Genes Dev 20: 2654-2659, 2006 https://doi.org/10.1101/gad.1457106

15. Zhou Y, Liu HX, Mistretta CM: Bone morphogenetic proteins and noggin: inhibiting and inducing fungiform taste 
papilla development. Dev Biol 297: 198-213, 2006.

https://doi.org/10.1016/j.ydbio.2006.05.022

16. Denny PC, Ball WD, Redman RS: Salivary glands: a paradigm for diversity of gland development. Crit Rev Oral Biol Med 8: 51-75, 1997. https://doi.org/10.1177/10454411970080010301

17. Chuong CM, Chodankar R, Widelitz RB, Jiang TX: Evo-devo of feathers and scales: building complex epithelial appendages. Curr Opin Genet Dev 10: 449-456, 2000. https://doi.org/10.1016/s0959-437x(00)00111-8

18. Chuong CM: The making of a feather: homeoproteins, retinoids and adhesion molecules. Bioessays 15: 513-521, 1993. https://doi.org/10.1002/bies.950150804

19. Antonio N: Ten Cate's oral histology: development, structure, and function. 7th ed. Mosby Elsevier, St. Louis, Missouri, pp.290-318, 2008.

20. Jeong SJ, Jeong MJ: Morphological study on the correlation of prenatal and postnatal development between mouse parotid salivary gland and tooth. Appl Microsc 47: 242-250, 2017. https://doi.org/10.9729/AM.2017.47.4.242

21. Bryk SG, Orlandini SZ, Gheri G, Sgambati E: Development of the chick tongue. A scanning electron microscopical investigation. Ann Anat 174: 531-534, 1992. https://doi.org/10.1016/S0940-9602(11)80317-1

22. Skieresz-Szewczyk K, Jackowiak H, Ratajczak M: LM and TEM study of the orthokeratinized and parakeratinized epithelium of the tongue in the domestic duck (Anas platyrhynchos f. domestica). Micron 67: 117-124, 2014. https://doi.org/10.1016/j.micron.2014.07.004

23. Skieresz-Szewczyk K, Jackowiak H, Kontecka $H$ : Morphogenesis of the tongue mucosa in the domestic duck (Anas platyrhynchos f. domestica) during the late embryonic stages. Microsc Res Tech 77: 667-674, 2014. https://doi.org/10.1002/jemt.22387

24. Hall JM, Hooper JE, Finger TE: Expression of sonic hedgehog, patched, and Gli1 in developing taste papillae of the mouse. J Comp Neurol 406: 143-155, 1999. https://doi.org/10.1002/(sici)1096-9861(19990405)406:2<14 3::aid-cne1>3.0.co;2-x

25. Tickle C: Vertebrate limb development. Curr Opin Genet Dev 5: 478-484, 1995. https://doi.org/10.1016/0959-437x(95)90052-i

26. Mbiene JP, Mistretta CM: Initial innervation of embryonic rat tongue and developing taste papillae: nerves follow distinctive and spatially restricted pathways. Acta Anat (Basel) 160: 139-158, 1997. https://doi.org/10.1159/000148006

27. Amasaki H, Arai R, Ogawa M, et al.: Postnatal development of the mouse volatile papilla taste bud cells. J Vet Med Sci 65: 541-543, 2003. https://doi.org/10.1292/jvms.65.541

28. Jung HS, Akita K, Kim JY: Spacing patterns on tongue surface-gustatory papilla. Int J Dev Biol 48: 157-161, 2004. https://doi.org/10.1387/ijdb.15272380

29. Iwasaki S, Yoshizawa H, Aoyagi H: Immunohistochemical expression of keratins 13 and 14 in the lingual epithelium of rats during the morphogenesis of filiform papillae. Arch Oral Biol 51: 416-426, 2006. https://doi.org/10.1016/j.archoralbio.2005.09.009

30. Iwasaki S, Okumura Y, Kumakura M: Ultrastructural study of the relationship between the morphogenesis of filiform papillae and the keratinization of the lingual epithelium in the mouse. Cells Tissues Organs 165: 91-103, 1999. https://doi.org/10.1159/000016679

31. Wojcik SM, Longley MA, Roop DR: Discovery of a novel murine keratin 6 (K6) isoform explains the absence of hair and nail defects in mice deficient for K6a and K6b. J Cell Biol 154: 619-630, 2001. https://doi.org/10.1083/jcb.200102079

32. Wong P, Colucci-Guyon E, Takahashi K, Gu C, Babinet C, Coulombe PA: Introducing a null mutation in the mouse K6alpha and K6beta genes reveals their essential structural role in the oral mucosa. J Cell Biol 150: 921-928, 2000. https://doi.org/10.1083/jcb.150.4.921 